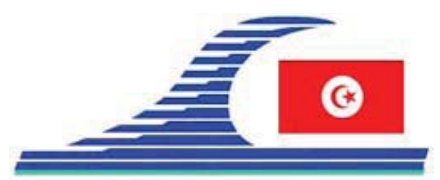

\title{
Simulation de l'interaction de la houle avec un obstacle submergé par une méthode de couplage Boussinesq - Equation intégrale
}

\author{
Mohammed HAMIDOU ${ }^{1}$, Mohamed KADRI ${ }^{2}$, Bernard MOLIN ${ }^{3}$, \\ Abdelkader TAHAKOURT ${ }^{4}$, Olivier KIMMOUN ${ }^{3}$
}

1. Département Hydraulique, Université de M'sila, Algérie.hamidou_moh@yahoo.fr

2. Département Génie Civil, Université de Boumerdès, Algérie.

3. Ec. Centrale Marseille \& IRPHE, 13451 Marseille cedex 20.bmolin@ec-marseille.fr

4. Département Génie Civil, Université de Béjaia, Algérie.

Résumé : On propose ici une méthode qui permet d'assurer un couplage fort entre le modèle Boussinesq (BINGHAM et al., 2009) et le modèle équation intégrale. Un cas test a été réalisé sur la propagation de la houle sur un obstacle submergé.

Mots-clés : Equations de Boussinesq - Equation intégrale - Bassin à houle numérique

\section{Introduction}

Parmi les différentes formulations des équations de Boussinesq développées ces dernières années, la formulation de MADSEN et al., (2002) en termes de vitesses, représente la formulation de Boussinesq la plus précise. JAMOIS et al., (2006) ont redérivé cette formulation, pour développer une formulation en terme de potentiel des vitesses, valable pour des profondeurs d'eau adimensionnelles $k h \approx 10$ en ce qui concerne les conditions de dispersion ( $k$ étant le nombre d'onde et $h$ la profondeur), tandis que pour les conditions cinématiques au sein du fluide la validité du modèle est réduite à $k h \approx 4$. BINGHAM et al., (2009) ont étendu la précision de ces propriétés jusqu'a $k h \approx 25$ pour la dispersion et $k h \approx 10$ pour la cinématique interne de l'écoulement. Les modèles cités ci-dessus permettent de simuler la propagation d'états de mer fortement non linéaires sur des bathymétries éventuellement (lentement) variables. Leur application à l'interaction de la houle avec des structures marines est restreinte à des obstacles immobiles à parois verticales. Une manière pour pouvoir traiter le cas d'obstacles de forme quelconque et de structures flottantes est de partitionner le domaine fluide en deux sous-domaines : un sous-domaine extérieur traité par un modèle Boussinesq et un sous-domaine proche traité par la méthode équation intégrale (E.I.). Dans ce présent travail, on propose une méthode qui permet d'assurer un couplage fort entre le modèle Boussinesq (BINGHAM et al., 2009) et le modèle (E.I.). La mise en œuvre et la validation du modèle sont réalisées.

\section{Théorie}

On utilise un système de coordonnées rectangulaires $O$ xz tel que l'axe x coïncide avec DOI: $10.5150 / \mathrm{cmcm} .2009 .009$ 
la surface libre au repos et que l'axe z soit vertical ascendant (Figure 1). Le fluide est supposé parfait, isovolume, et l'écoulement irrotationnel. Soit $\phi(x, z, t)$ le potentiel des vitesses et $\eta(x, t)$ l'élévation de la surface libre. Suivant ZAHKAROV (1986) on écrit les conditions, dynamique et cinématique, de la surface libre sous la forme :

$\widetilde{\phi}_{t}=-g \eta+\frac{\widetilde{w}^{2}}{2}\left(1+\eta_{x}^{2}\right)-\frac{1}{2} \widetilde{\phi}_{x}^{2}$

$\eta_{t}=\widetilde{w}\left(1+\eta_{x}^{2}\right)-\eta_{x} \widetilde{\phi}_{x}$

où $\widetilde{\phi}(x, t)=\phi(x, \eta(x, t), t)$ et $\widetilde{w}(x, t)=\phi_{z}(x, \eta(x, t), t)$ sont respectivement le potentiel et la vitesse verticale de la surface libre. Le potentiel $\phi$ vérifie aussi les conditions $\Delta \phi=0$ dans le domaine fluide et $\partial \phi / \partial n=0$ sur les frontières rigides. L'intégration des équations (1) et (2) nécessite la détermination de la vitesse verticale $\widetilde{w}$.

Dans le domaine Boussinesq, dans le cas ou la profondeur $h$ est supposée constante, suivant la formulation de (BINGHAM et al., 2009), le potentiel des vitesses $\phi(x, z, t)$ et la vitesse verticale $w(x, z, t)$ sont développés sous la forme :

$$
\begin{gathered}
\phi(x, z, t)=\left(\hat{\phi}^{*}+\frac{\hat{z}^{2}}{18} \hat{\phi}_{x x}^{*}+\frac{\hat{z}^{4}}{504} \hat{\phi}_{x x x x}^{*}\right)+\left(\hat{w}^{*}+\frac{\hat{z}^{2}}{18} \hat{w}_{x x}^{*}+\frac{\hat{z}^{4}}{504} \hat{w}_{x x x}^{*}\right) \psi+\left(-\frac{1}{2} \hat{\phi}_{x x}^{*}-\frac{\hat{z}^{2}}{36} \hat{\phi}_{x x x x}^{*}\right) \\
\psi^{2}+\left(-\frac{1}{6} \hat{w}_{x x}^{*}-\frac{\hat{z}^{2}}{108} \hat{w}_{x x x x}^{*}\right) \psi^{3}+\left(\frac{1}{24} \hat{\phi}_{x x x x}^{*}\right) \psi^{4}+\left(\frac{1}{120} \hat{w}_{x x x x}^{*}\right) \psi^{5} \\
w(x, y, z, t)=-\psi \hat{\phi}_{x x}^{*}-\left(-\frac{\psi^{3}}{6}+\frac{\psi \hat{z}^{2}}{18}\right) \hat{\phi}_{x x x x}^{*}-\left(\frac{\psi^{5}}{120}-\frac{\psi^{3} \hat{z}^{2}}{108}+\frac{\psi \hat{z}^{4}}{504}\right) \hat{\phi}_{x x x x x}^{*}+\hat{w}^{*}+ \\
\left(-\frac{\psi^{2}}{2}+\frac{\hat{z}^{2}}{18}\right) \hat{w}_{x x}^{*}+\left(\frac{\psi^{4}}{24}-\frac{\psi^{2} \hat{z}^{2}}{36}+\frac{\hat{z}^{4}}{504}\right) \hat{w}_{x x x x}^{*}
\end{gathered}
$$

Avec $\psi=(z-\hat{z}) ; \hat{z}=-h / 2 ; \hat{\phi}^{*}(x, t)$ et $\hat{w}^{*}(x, t)$ sont des variables utilitaires (voir JAMOIS (2005) et BINGHAM et al., (2009)).

Dans le domaine Equation intégrale en applique la seconde identité de Green

$$
\alpha(p) \phi(p)+\int_{\Gamma} \phi(q) \nabla_{q} G(p, q) \cdot \vec{n}_{q} d \Gamma_{q}=\int_{\Gamma} G(p, q) \nabla_{q} \phi(q) \cdot \vec{n}_{q} d \Gamma_{q}
$$

La fonction de Green $G(p, q)$ est ici simplement $\ln (p, q)$. $\alpha(p)$ est l'angle entre les deux tangentes au contour en $\mathrm{P}$, la normale $\vec{n}$ est dirigé vers l'extérieur du domaine. La résolution de cette équation (COINTE, 1990), nous donne la vitesse verticale dans le domaine E.I.

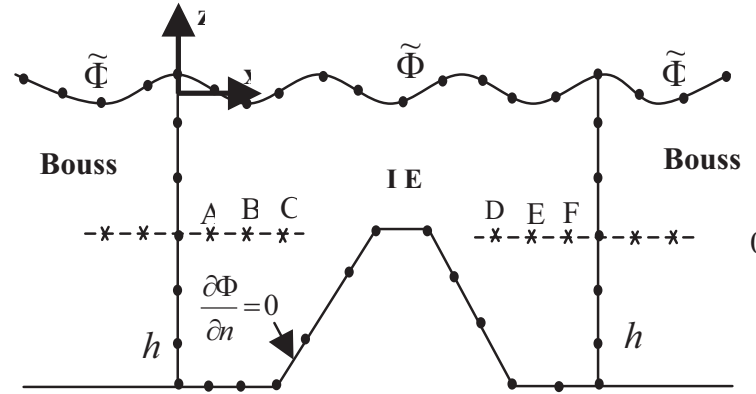

Figure 1. Définition géométrique.

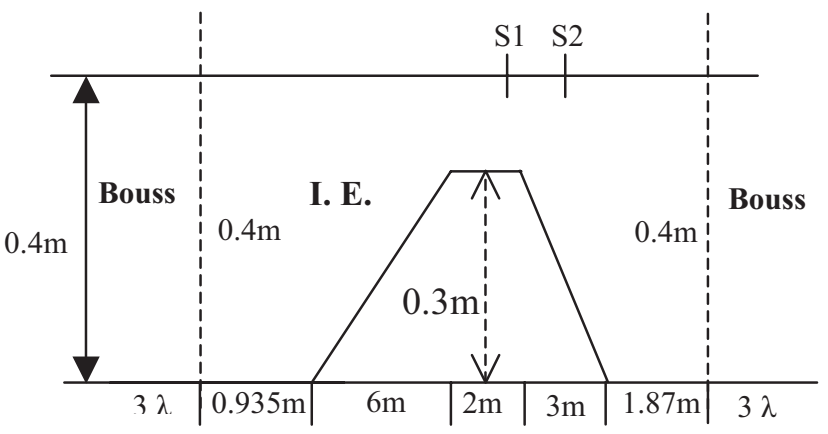

Figure 2. Positions des sondes 


\section{Méthode de couplage}

La résolution du problème fluide dans le domaine Boussinesq nécessite l'introduction de points additionnels à l'intérieur du domaine E. I., soient $\mathrm{A}, \mathrm{B}, \mathrm{C}, \mathrm{D}, \mathrm{E}$ et $\mathrm{F}$ où les valeurs de $\hat{\phi}_{A}^{*}, \hat{\phi}_{B}^{*}, \hat{\phi}_{C}^{*}, \hat{\phi}_{D}^{*}, \hat{\phi}_{E}^{*}, \hat{\phi}_{F}^{*}, \hat{w}_{A}^{*}, \hat{w}_{B}^{*}, \hat{w}_{C}^{*}, \hat{w}_{D}^{*}, \hat{w}_{E}^{*}$ et $\hat{w}_{F}^{*}$ sont requises (voir figure 1). Il s'agit de raccorder, sur les segments verticaux $x=0$ et $\mathrm{x}=\mathrm{L},-h \leq z \leq \eta$, les potentiels et les vitesses horizontales. On va donc exprimer, dans le domaine E. I., à la frontière, le potentiel et la vitesse horizontale suivant la même forme polynomiale que dans le domaine Boussinesq.

Dans le domaine E.I, le potentiel est maintenant écrit sous la forme :

$$
\phi(x, z, t)=\varphi_{p}(x, z, t)+\sum_{i=1}^{12} A_{i} \varphi_{i}(x, z, t)
$$

Le potentiel $\varphi_{p}$ vérifie l'équation de Laplace $\Delta \varphi_{p}=0$ dans le domaine E. I., la condition à la surface libre $\varphi_{p}=\widetilde{\phi}$, la condition $\partial \varphi_{p} / \partial n=0$ sur les frontières solides et la condition $\partial \varphi_{p} / \partial x=0$ sur les frontières $x=0$ et $x=L$.

Les potentiels $\varphi_{i}$ vérifient l'équation de Laplace $\Delta \varphi_{i}=0$ dans le domaine E. I., la condition à la surface libre $\varphi_{i}=0$, la condition $\partial \varphi_{i} / \partial n=0$ sur les frontières solides et les conditions :

$$
\begin{array}{llll}
\text { pour } \mathrm{i}=1,6: \frac{\partial \varphi_{i}}{\partial x}=(z-\hat{z})^{i-1} & , x=0 & \text { et } \frac{\partial \varphi_{i}}{\partial x}=0 & , x=L \\
\text { pour } \mathrm{i}=7,12: \frac{\partial \varphi_{i}}{\partial x}=0 & , x=0 & \text { et } \frac{\partial \varphi_{i}}{\partial x}=(z-\hat{z})^{i-7} & , x=L
\end{array}
$$

On a donc écrit, d'une part, les vitesses horizontales $\phi_{x}(0, z, t)$ et $\phi_{x}(L, z, t)$ sous la forme $\phi_{x}(0, z, t)=\sum_{i=1}^{6} A_{i}(z-\hat{z})^{i-1}, \quad \phi_{x}(L, z, t)=\sum_{i=7}^{12} A_{i}(z-\hat{z})^{i-7}$

et, d'autre part, les potentiels $\phi(0, z, t)$ et $\phi(L, z, t)$ sous la forme polynomiale :

$$
\phi(0, z, t)=\sum_{i=1}^{6}(z-\hat{z})^{i-1}\left(\alpha_{p i}+\sum_{j=1}^{12} \alpha_{i j} A_{j}\right), \phi(L, z, t)=\sum_{i=1}^{6}(z-\hat{z})^{i-1}\left(\beta_{p i}+\sum_{j=1}^{12} \beta_{i j} A_{j}\right)
$$

Les coefficients $\alpha_{p j}, \alpha_{i j}, \beta_{p j}$ et $\beta_{i j}$ sont déterminés par une méthode des moindres carrés.

L'identification des relations (7) et (8) avec les développements polynomiaux du potentiel et de la vitesse horizontale dans le domaine Boussinesq, nous permet de résoudre le problème fluide dans le domaine Boussinesq et de déterminer la vitesse verticale de la surface libre. On peut alors avancer en temps $\widetilde{\phi}$ et $\eta$, ici par un schéma Runge-Kutta d'ordre 4.

\section{Applications}

On considère le cas de propagation de la houle sur une barre immergée figure (2), ce cas test est tiré du rapport de mesures expérimentales et numériques de Dingemans (1994- 
cas test $\mathrm{A}$ ). Les sondes de mesure sont localisées $7.5 \mathrm{~m}$ et $8.5 \mathrm{~m}$ à partir du début de la pente amont de la barre. La profondeur d'eau varie de $40 \mathrm{~cm}$ à $10 \mathrm{~cm}$. On considère le cas d'une houle régulière de période égale à $2.02 \mathrm{~s}$, d'amplitude égale à $1 \mathrm{~cm}$, et de longueur d'onde $\lambda$ égale à $3.741 \mathrm{~m}$.

Les courbes de la figure 3 montrent un bon accord entre les résultats numériques et les résultats expérimentaux tirés du cas test A du rapport de DINGEMANS (1994).
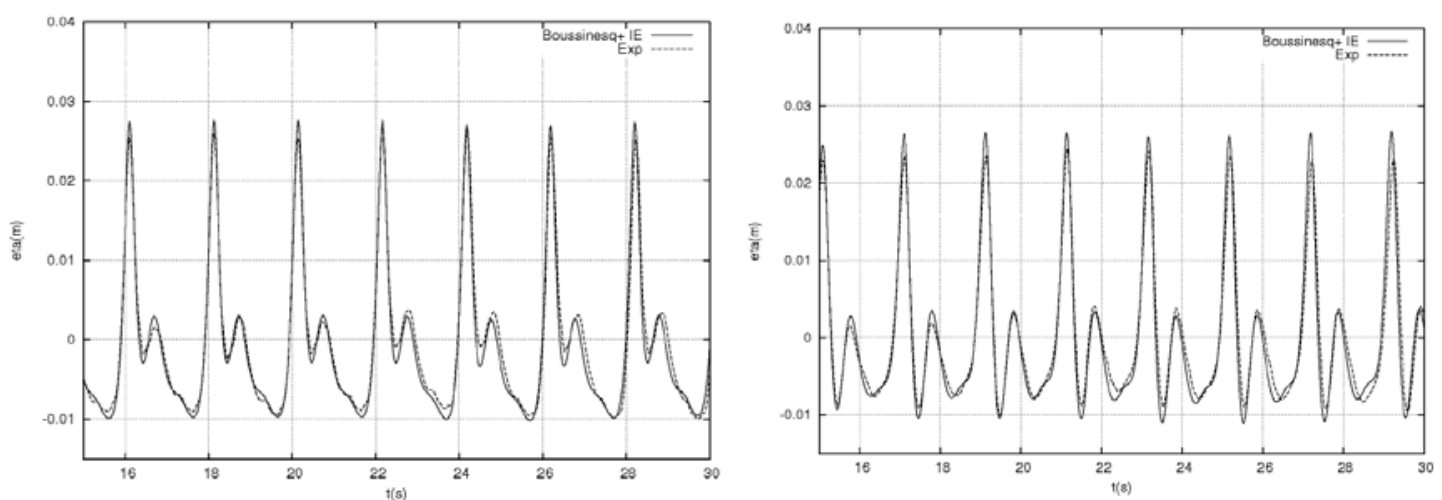

Figure 3. Évolution temporelle de la surface libre au niveau des sondes S1 à gauche et $S 2$ à droite.

\section{Conclusion}

On a proposé un modèle de couplage entre les équations de Boussinesq étendues et la méthode équation intégrale, les résultats obtenus par la simulation de la houle sur une barre immergée présentent un bon accord avec les résultats expérimentaux. L'extension du modèle au cas de structures flottantes est simple à réaliser. La généralisation du modèle aux problèmes à 3 dimensions est envisageable.

\section{Références bibliographiques}

BINGHAM H.B., MADSEN P.A., FUHRMAN, D.R. (2009). Velocity potential formulations of highly accurate Boussinesq-type models. Coastal Engineering. 56, pp 467-478.

COINTE R. (1990). Numerical simulation of a wave channel. Engineering analysis with Boundary Elements, 7, pp 167-177.

DINGEMANS M.W. (1994). Comparison of computations with Boussinesq-like models and laboratory measurement. MAST Report G8-M.

JAMOIS E. (2005). Interaction houle structure en zone côtière. Thèse de doctorat, AixMarseille II.

MADSEN P.A., BINGHAM H.B., LIU H. (2002). A new Boussinesq method for fully nonlinear waves from shallow to deep water. J. Fluid Mech. 462, pp 1-30.

ZAKHAROV V.E. (1968). Stability of periodic waves of finite amplitude on the surface of a deep fluid. J. Appl. Mech. Tech. Phys. 9, pp 190-194. 